Graham C. Holt

\title{
Carbon neutrality—what does it mean?
}

ABSTRACT. The achievement of carbon neutrality has become an inescapable feature of the news media and like most long-running news stories, the momentum of the reporting often loses the underlying facts as the tale unfolds in everyday exposure. This article seeks to restore some of those facts and questions the assumptions and direction that politicians and the press (the two seem inextricably linked these days so that it is often difficult to know who leads who) are taking us in the quest to avert anthropogenic-related climate change. A case for carbon neutrality achieved through carbon trading, rather than politicians posturing on the world stage for national carbon emission reduction, is made on the basis that whether or not man is critically affecting climate, at least a global mechanism is thereby in place to cope.

Nanotechnology Perceptions 5 (2009) 135-145

Nonsubscribers: purchase individual article 